\title{
Lifetime measurement of neutron-rich even-even molybdenum isotopes
}

D. Ralet,,${ }^{1,2,3,{ }^{*}}$ S. Pietri, ${ }^{2}$ T. Rodríguez, ${ }^{4}$ M. Alaqeel,${ }^{5}$ T. Alexander, ${ }^{6}$ N. Alkhomashi, ${ }^{7}$ F. Ameil, ${ }^{2}$ T. Arici,,${ }^{2,8}$ A. Ataç, ${ }^{9,10}$ R. Avigo, ${ }^{11}$ T. Bäck, ${ }^{9}$ D. Bazzacco, ${ }^{12}$ B. Birkenbach, ${ }^{13}$ P. Boutachkov, ${ }^{2}$ B. Bruyneel,,${ }^{14}$ A. M. Bruce, ${ }^{15}$ F. Camera,,${ }^{11}$ B. Cederwall, ${ }^{9}$ S. Ceruti, ${ }^{11}$ E. Clément, ${ }^{16}$ M. L. Cortés, ${ }^{1,2}$ D. Curien, ${ }^{17}$ G. De Angelis, ${ }^{12}$ P. Désesquelles, ${ }^{3}$ M. Dewald,${ }^{13}$ F. Didierjean, ${ }^{17}$ C. Domingo-Pardo, ${ }^{18}$ M. Doncel,${ }^{9}$ G. Duchêne,,${ }^{17}$ J. Eberth, ${ }^{13}$ A. Gadea, ${ }^{18}$ J. Gerl, ${ }^{2}$ F. Ghazi Moradi, ${ }^{9}$ H. Geissel,${ }^{2}$ T. Goigoux,${ }^{2}$ N. Goel,${ }^{2}$ P. Golubev, ${ }^{19}$ V. González,${ }^{20}$ M. Górska, ${ }^{2}$ A. Gottardo, ${ }^{12}$ E. Gregor, ${ }^{1,2}$ G. Guastalla, ${ }^{1,2}$ A. Givechevv ${ }^{1,2}$ T. Habermann,,${ }^{1,2}$ M. Hackstein, ${ }^{13}$ L. Harkness-Brennan, ${ }^{21}$ G. Henning, ${ }^{2}$ H. Hess,${ }^{13}$ T. Hüyük,${ }^{18}$ J. Jolie, ${ }^{13}$ D. S. Judson, ${ }^{21}$ A. Jungclaus, ${ }^{22}$ R. Knoebel, ${ }^{2}$ I. Kojouharov, ${ }^{2}$ A. Korichi ${ }^{3}{ }^{2}$ W. Korten,${ }^{23}$ N. Kurz, ${ }^{2}$ M. Labiche, ${ }^{24}$ N. Lalović,,${ }^{2,19}$ C. Louchart-Henning, ${ }^{1,2}$ D. Mengoni, ${ }^{12,25}$ E. Merchán, ${ }^{1,2}$ B. Million, ${ }^{11}$ A. I. Morales, ${ }^{11}$ D. Napoli, ${ }^{26}$ F. Naqvi, ${ }^{2}$ J. Nyberg, ${ }^{27}$ N. Pietralla ${ }^{1,2}$ Zs. Podolyák, ${ }^{6}$ A. Pullia, ${ }^{11,28}$ A. Prochazka, ${ }^{2}$ B. Quintana ${ }^{29}$ G. Rainovski, ${ }^{30}$ M. Reese,,${ }^{1,2}$ F. Recchia, ${ }^{12,25}$ P. Reiter ${ }^{13}$ D. Rudolph, ${ }^{19}$ M. D. Salsac, ${ }^{14}$ E. Sanchis, ${ }^{20}$ L. G. Sarmiento, ${ }^{19}$ H. Schaffner, ${ }^{2}$ C. Scheidenberger, ${ }^{2}$ L. Sengele 17 B. S. Nara Singh, ${ }^{31}$ P. P. Singh, ${ }^{1,2}$ C. Stahl,,${ }^{1}$ O. Stezowski, ${ }^{32}$ P. Thoele, ${ }^{13}$ J. J. Valiente Dobon, ${ }^{12}$ H. Weick, ${ }^{2}$ A. Wendt, ${ }^{13}$ O. Wieland, ${ }^{11}$ J. S. Winfield, ${ }^{2}$ H. J. Wollersheim, ${ }^{2}$ and M. Zielinska ${ }^{23}$ (PreSPEC and AGATA Collaborations)

${ }^{1}$ Institut für Kernphysik, Technisch Universität Darmstadt, D-64289 Darmstadt, Germany

${ }^{2}$ Helmholtzzentrum für Schwerionenforschung GmbH (GSI), D-64291 Darmstadt, Germany

${ }^{3}$ CSNSM, Univ. Paris-Sud, CNRS/IN2P3, Universitié Paris-Saclay, F-91405 Orsay, France

${ }^{4}$ Departamento de Francesica Teorica, Universidad Autonoma de Madrid, E-28049 Madrid, Spain

${ }^{5}$ Department of Physics, College of Sciences, Al Imam Mohammad Ibn Saud Islamic University (IMSIU), P.O. Box 90950, Riyadh 11623, Saudi Arabia

${ }^{6}$ Department of Physics, University of Surrey, Guidford, GU-27XH, United Kingdom

${ }^{7}$ King Abdulaziz City for Science and Technology (KACST), P.O. Box 6086, Riyadh 11442, Saudi Arabia

${ }^{8}$ Justus-Liebig-Universität Giessen, D-35392 Giessen, Germany

${ }^{9}$ Department of Physics, Royal Institute of Technology (KTH), SE-10691 Stockholm, Sweden

${ }^{10}$ Department of Physics, Faculty of Science, Ankara University, TR-06100 Tandoğan, Ankara, Turkey

${ }^{11}$ Istituto Nazionale di Fisica Nucleare Sezione di Milano, IT-20133 Milano, Italy

${ }^{12}$ Istituto Nazionale di Fisica Nucleare, Sezione di Padova, IT-35131 Padova, Italy

${ }^{13}$ Institut für Kernphysik, Universität zu Köln, D-50937 Köln, Germany

${ }^{14}$ Institut de Recherche sur les lois Fondamentales de l'Univers IRFU, CEA/DSM, Centre CEA de Saclay, F-91191 Gif-sur-Yvette Cedex, France

${ }^{15}$ School of Computing, Engineering and Mathematics, University of Brighton, Brighton, BN2 4GJ, United Kingdom

${ }^{16}$ Grand Accélérateur National d'Ions Lourds GANIL, CEA/DSAM and CNRS/IN2P3, BP 55027, F-14076 CAEN Cedex 05, France

${ }^{17}$ IPHC/CNRS-University of Strasbourg, F-67037 Strasbourg, France

${ }^{18}$ Instituto de Física Corpuscular, CSIC-Universidad de Valencia, E-46071 Valencia, Spain

${ }^{19}$ Department of Physics, Lund University, SE-22100 Lund, Sweden

${ }^{20}$ Departamento de Ingeniera Electrónica, Universitat de Valencia, Burjassot, E-46100 Valencia, Spain

${ }^{21}$ Oliver Lodge Laboratory, The University of Liverpool, Liverpool, L69 7ZE, United Kingdom

${ }^{22}$ Instituto de Estructura de la Materia, CSIC Madrid, E-28006 Madrid, Spain

${ }^{23}$ Institut de Recherche sur les lois Fondamentales de l'Univers (IRFU), CEA/DSM, Centre CEA de Saclay, F-91191 Gif-sur-Yvette Cedex, France

${ }^{24}$ STFC Daresbury Laboratory, Daresbury, Warrington, WA4 4AD, United Kingdom

${ }^{25}$ Dipartimento di Fisica e Astronomia dell'Universit di Padova, I-35131 Padova, Italy

${ }^{26}$ Laboratori Nazionali di Legnaro, INFN, I-35020 Legnaro (PD), Italy

${ }^{27}$ Department of Physics and Astronomy, Uppsala University, SE-75120 Uppsala, Sweden

${ }^{28}$ University of Milano, Department of Physics, I-20133 Milano, Italy

${ }^{29}$ Laboratorio de Radiaciones Ionizantes, Universidad de Salamanca, E-37008 Salamanca, Spain

${ }^{30}$ Faculty of Physics, St. Kliment Ohridski University of Sofia, BG-1164 Sofia, Bulgaria

${ }^{31}$ Department of Physics, University of York, York YO10 5DD, United Kingdom

${ }^{32}$ Université de Lyon, CNRS-IN2P3, Institut de Physique Nucléaire de Lyon, F-69622 Villeurbanne, France

(Received 9 November 2016; published 27 March 2017)

Background: In the neutron-rich $A \approx 100$ mass region, rapid shape changes as a function of nucleon number as well as coexistence of prolate, oblate, and triaxial shapes are predicted by various theoretical models. Lifetime measurements of excited levels in the molybdenum isotopes allow the determination of transitional quadrupole moments, which in turn provides structural information regarding the predicted shape change.

*ralet@csnsm.in2p3.fr 
Purpose: The present paper reports on the experimental setup, the method that allowed one to measure the lifetimes of excited states in even-even molybdenum isotopes from mass $A=100$ up to mass $A=108$, and the results that were obtained.

Method: The isotopes of interest were populated by secondary knock-out reaction of neutron-rich nuclei separated and identified by the GSI fragment separator at relativistic beam energies and detected by the sensitive PreSPECAGATA experimental setup. The latter included the Lund-York-Cologne calorimeter for identification, tracking, and velocity measurement of ejectiles, and AGATA, an array of position sensitive segmented HPGe detectors, used to determine the interaction positions of the $\gamma$ ray enabling a precise Doppler correction. The lifetimes were determined with a relativistic version of the Doppler-shift-attenuation method using the systematic shift of the energy after Doppler correction of a $\gamma$-ray transition with a known energy. This relativistic Doppler-shiftattenuation method allowed the determination of mean lifetimes from 2 to 250 ps.

Results: Even-even molybdenum isotopes from mass $A=100$ to $A=108$ were studied. The decays of the low-lying states in the ground-state band were observed. In particular, two mean lifetimes were measured for the first time: $\tau=29.7_{-9.1}^{+11.3}$ ps for the $4^{+}$state of ${ }^{108}$ Mo and $\tau=3.2_{-0.7}^{+0.7}$ ps for the $6^{+}$state of ${ }^{102}$ Mo.

Conclusions: The reduced transition strengths $B(E 2)$, calculated from lifetimes measured in this experiment, compared to beyond-mean-field calculations, indicate a gradual shape transition in the chain of molybdenum isotopes when going from $A=100$ to $A=108$ with a maximum reached at $N=64$. The transition probabilities decrease for ${ }^{108}$ Mo which may be related to its well-pronounced triaxial shape indicated by the calculations.

DOI: 10.1103/PhysRevC.95.034320

\section{INTRODUCTION}

In the nuclear landscape, the neutron-rich mass region $A \approx 100$ located between the major shells $28<Z<50$ and $50<N<82$ is known to exhibit strongly deformed nuclei with a quadrupole deformation parameter $\beta_{2}$ ranging from 0.3 to 0.4 [1]. The neutron-rich strontium $(Z=38)$ and zirconium $(Z=40)$ isotopes exhibit a drastic shape change from spherical shape at $N \leqslant 58$ to strongly deformed at $N \geqslant 60$. The neutron-rich molybdenum $(Z=42)$ isotopes follow a similar trend, with a spherical shape at $N=56$ and a smooth increase of the quadrupole deformation toward $N=64$. The less rapid shape evolution in the molybdenum isotopes, compared to neighboring zirconium isotopes was suggested by Rodríguez-Guzmán [2] as resulting from emerging triaxiality. At $N=68,{ }^{110} \mathrm{Mo}$ is predicted [3] to be more oblate deformed than the transitional nucleus ${ }^{108}$ Mo which is foreseen triaxial [3]. Experimental evidence for triaxiality in neutron-rich molybdenum isotopes was reported in Refs. [4-6] for even-even nuclei and in Ref. [7] for the odd-even ones. Hua [8] suggests that a pair alignment in the $h_{11 / 2}$ neutron orbital is responsible for the triaxiality observed in the molybdenum isotopes.

In this midshell region, the shell-model valence space is too large to calculate observables such as excitation energies and electromagnetic matrix elements. To interpret observations, the interacting boson model $[9,10]$ or beyondmean-field (BMF) calculations [11-13] can be used. In this paper, we present new results of BMF calculations with the D1S parametrization of the Gogny interaction [14] and the symmetry conserving configuration mixing (SCCM) [15] approach.

The production and study of these neutron-rich isotopes are made possible using various techniques. Prompt $\gamma$-ray spectroscopy of spontaneous fission fragments $[5,7,16,17]$ was shown to be a powerful technique to study nuclei up to ${ }^{106} \mathrm{Mo}$. To reach more neutron-rich isotopes in this region, high energy in-flight fission was used. For example, $\beta$-delayed $\gamma$-ray spectroscopy using a radioactive ion beam (RIB) allowed spectroscopic information up to ${ }^{110} \mathrm{Mo}$ [6] to be obtained. Because of the refractory character of molybdenum, radioactive beams of this element cannot be delivered from ISOL facilities, which makes direct measurements of $B(E 2)$ transition strengths via low-energy Coulomb excitation impossible. The alternative is to measure lifetimes of excited states, which, for example, was achieved with a plunger technique following the spontaneous fission of ${ }^{248} \mathrm{Cm}$ [5]. The present paper presents a variant of the relativistic Doppler-shift-attenuation method and its application to the picosecond lifetimes in neutron-rich Mo isotopes.

The measurement described here was performed via inbeam $\gamma$-ray spectroscopy of nuclei produced in a two-step reaction process. The first reaction at the entrance of a separator produced ${ }_{43}^{109} \mathrm{Tc}$ ions. The neutron-rich molybdenum isotopes were created when this radioactive incoming beam underwent a proton and $x$ neutrons knock-out at a secondary target. Following the secondary reaction, the prompt $\gamma$ rays from excited molybdenum isotopes were detected with the Advanced GAmma Tracking Array (AGATA) [18]. The experimental setup, in which AGATA was for the first time used with a radioactive ion beam [19] is described in Sec. II. The data analysis is detailed in Sec. III. The combination of several detectors provided a unique experimental setup for lifetime studies. The method developed for the lifetime determination is described in Sec. IV and the results are compared to literature values. A full comparison of the reduced transition strengths, $B\left(E 2 ; J_{i} \rightarrow J_{f}\right)$, with those calculated with the BMF approach is performed in Sec. V. Conclusions and outlook are given in Sec. VI.

\section{EXPERIMENTAL APPROACH}

The universal linear accelerator (UNILAC) at GSI preaccelerated a beam of ${ }^{238} \mathrm{U}$ ions which was injected into the SIS-18 synchrotron [20]. The uranium ions were further 


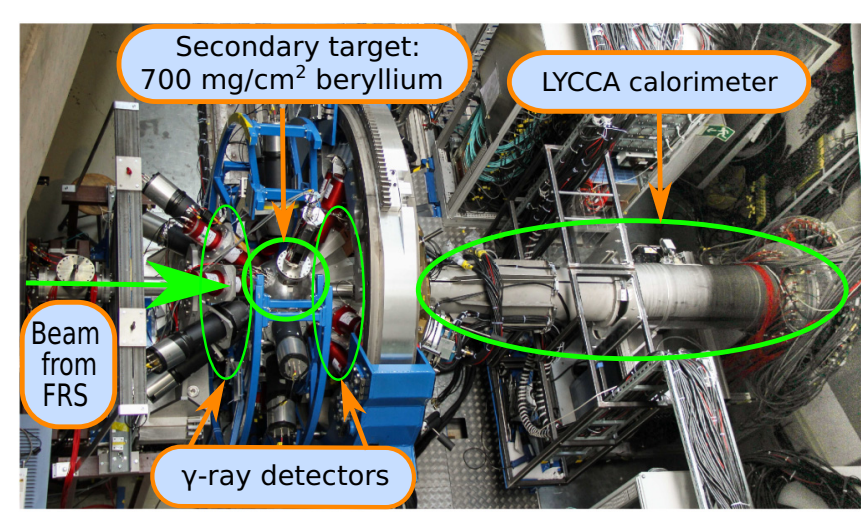

FIG. 1. Photograph of the experimental setup positioned behind the last FRS plastic detector.

accelerated by SIS-18 up to an energy of $600 \mathrm{MeV} / \mathrm{u}$. The slow extraction of SIS-18 was used with a cycle time of four seconds to maximize the intensity. The uranium beam impinged and fissioned on a $1033 \mathrm{mg} / \mathrm{cm}^{2}$ beryllium production target at the entrance of the fragment separator (FRS) [21]. Before the production target, the beam intensity was measured by a secondary electron transmission monitor (SEETRAM) [22] and reached a steady $2 \times 10^{9}$ particles per synchrotron cycle. The FRS was tuned for monoenergetic mode [21] to select ${ }^{109} \mathrm{Tc}$. The standard FRS detector suite was used for the identification of ions in the second stage of the FRS as described in Ref. [23]. The fission products reached a secondary $\sim 700-\mathrm{mg} / \mathrm{cm}^{2}$ thick beryllium target to undergo knock-out and enable in-beam $\gamma$-ray spectroscopy. The energy at the secondary target was reduced to $\sim 150 \mathrm{MeV} / \mathrm{u}$, using a degrader in the final focal plane of the FRS. The energy was chosen as a compromise between a high beam energy that allowed for a thick target, thus higher production yield, and the need to keep the photon background induced by the beam low, as presented in Ref. [24]. This background is attributed to bremsstrahlung in the target and surrounding detectors. It made the measurements of $\gamma$-ray peaks below $200 \mathrm{keV}$ infeasible (see below).

Slits in the horizontal and vertical directions constrained the incoming beam on the $5 \times 5 \mathrm{~cm}^{2}$ target. Three different settings of $B \rho$ were used. The first one, an isomer setting, was used to confirm the FRS particle identification. Detailed information on this type of setting can be found in Ref. [23].
The second and third settings were production settings in which ${ }^{109} \mathrm{Tc}$ and ${ }^{107} \mathrm{Nb}$, respectively, were centered on the secondary target. The monoenergetic mode assured that in both production settings ${ }^{108}$ Mo nuclei were also centered onto the target.

The total intensity measured by a plastic scintillator positioned at the last focal plane of the FRS was about 1900 particles per synchrotron cycle. The ${ }^{109} \mathrm{Tc}$ beam underwent a fragmentation in the secondary target to produce, e.g., ${ }^{108} \mathrm{Mo}$ in a one-proton knockout reaction. The fragments produced in this second-step fragmentation were tracked and identified by the Lund-York-Cologne calorimeter (LYCCA) [25]. The secondary target was surrounded by two $\gamma$-ray detector arrays: the high-purity-germanium detectors array AGATA [18] and the scintillator high-energy $\gamma$-ray detector (HECTOR+) [26]. The AGATA detectors were covering forwarded angle from $20^{\circ}$ to $60^{\circ}$. The HECTOR detectors were mainly positioned at backward angles. A photograph of the experimental setup is shown in Fig. 1.

The total number of events recorded in the experiment are given in Table I for different triggers. In the table, the trigger number refers to the type of coincidence requested in the experiment. T10 corresponds to the number of particles hitting the last FRS plastic scintillator, scaled down by a factor of $2^{8}$ to avoid losses caused by the dead time. T9 represents the trigger requesting a coincidence between a particle detected at the final focal plane of the FRS, a $\gamma$ ray detected by AGATA, and a particle reaching the last plasticscintillator detector of LYCCA. T8, quoted for completeness, is a coincidence between the FRS plastic scintillator, LYCCA, and the HECTOR + array of scintillator $\gamma$-ray detectors. More details on the trigger system can be found in Ref. [23]. In this experiment, an energy threshold on the $\gamma$-ray trigger request was set at $\sim 100 \mathrm{keV}$ for the AGATA detectors and $\sim 250$ $\mathrm{keV}$ for the HECTOR+detectors. The coincidence trigger rate reached on average $300 \mathrm{~Hz}$.

\section{FRAGMENT IDENTIFICATION}

The identification of the fragments in the FRS was performed using standard methods described in Ref. [21]. The detector layout can be found in Fig. 1 of Ref. [27]. The time of flight (ToF) of the fragments was measured with two plastic scintillators positioned at the intermediate and at the final focal plane of the FRS. The ToF provided the measurement of the

TABLE I. The number of events that were recorded in the experiment presented in this paper. The events that were properly identified at the final focal plane are indicated with particle identification (PID). The ratio refers to the number of selected identified ions in the setting divided by the total number of identified ions. The trigger number corresponds to the type of coincidence requested in this experiment (see text and Ref. [23] for additional details).

\begin{tabular}{|c|c|c|c|c|c|c|c|c|c|c|}
\hline \multirow[t]{2}{*}{$\begin{array}{l}\text { Setting } \\
\text { number }\end{array}$} & \multirow[t]{2}{*}{$\begin{array}{l}\text { Centered } \\
\text { nucleus }\end{array}$} & \multicolumn{6}{|c|}{ Number of triggers } & \multicolumn{3}{|c|}{$\begin{array}{c}\text { Ratio (\%) } \\
\text { of events with a valid PID }\end{array}$} \\
\hline & & $\begin{array}{l}\text { Trigger number } \\
\text { T8, T9, T10 }\end{array}$ & T9 & $\mathrm{T} 10$ & T8, T9, T10 & T9 & $\mathrm{T} 10$ & $\begin{array}{l}\text { Identi } \\
{ }^{109} \mathrm{Tc}\end{array}$ & $\begin{array}{l}\text { d nucleu } \\
{ }^{108} \mathrm{Mo}\end{array}$ & ${ }^{107} \mathrm{Nb}$ \\
\hline 2 & ${ }^{109} \mathrm{Tc}$ & $8.11 \times 10^{6}$ & $7.55 \times 10^{6}$ & $1.41 \times 10^{5}$ & $5.90 \times 10^{6}$ & $5.51 \times 10^{6}$ & $1.01 \times 10^{5}$ & 33.0 & 10.8 & 1.46 \\
\hline 3 & ${ }^{107} \mathrm{Nb}$ & $1.27 \times 10^{7}$ & $1.18 \times 10^{7}$ & $2.10 \times 10^{5}$ & $8.65 \times 10^{6}$ & $8.07 \times 10^{6}$ & $1.39 \times 10^{5}$ & - & 41.7 & 7.1 \\
\hline
\end{tabular}




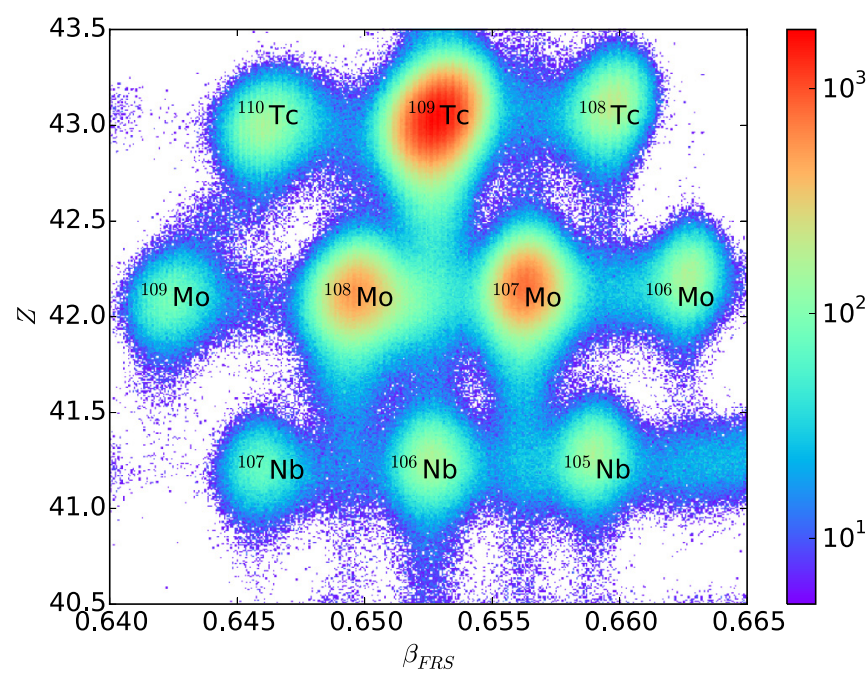

FIG. 2. Identification plot of species selected by the FRS for the setting centered on ${ }^{109} \mathrm{Tc}$. The color bar on the right-hand side indicates the number of counts per bin.

beam velocity by

$$
\beta_{\mathrm{FRS}}=\frac{L_{1} / \mathrm{ToF}_{\mathrm{FRS}}}{c},
$$

with the distance between the two plastic scintillators $L_{1}=$ $36.664 \mathrm{~m}$. The charge of the ions was measured with a multisampling-ionization chamber [28] (MUSIC) positioned at $988 \mathrm{~mm}$ behind the last dipole of the FRS. Positions and angles at the final focal plane were measured by two time-projection chambers [29] (TPCs) positioned at 91 and $1518 \mathrm{~mm}$ behind the exit window of the last dipole. Similarly, two TPCs were positioned at the intermediate focal plane at 286 and $1376 \mathrm{~mm}$ behind the middle focal plane degrader. In this experiment, the monoenergetic mode of the FRS allowed one to identify the fragments with the beam velocity and the charge number $Z$ as shown in Fig. 2. This identification provided three times more identified ions at the focal plane compared to when the conventional method for fragment separators was used with the same data [21]. The number of properly identified species are given in Table I. From that table, it can be noticed that it was possible to identify $\approx 70 \%$ of the recorded events.

The identification of the reaction products after the secondary target was done by LYCCA [25]. It comprised $16 \Delta E-E$ telescopes of double-sided-silicon-strip detectors (DSSSDs) for the measurement of the energy loss combined with cesium-iodide crystals (CsI) for the measurement of the total kinetic energy (TKE) as well as two circular plastic scintillators, or membranes, surrounded by 32 photomultiplier tubes (PMTs). To measure ejectile velocity independent of the interaction position of the particle onto the LYCCA plastic membrane [30], each individual PMT time was made independent of the distance of the interaction position to the PMT. The mean aligned time of the 32 PMTs was finally corrected for long-term shifts on an hourly basis. The time average of the 32 PMTs was used as the intrinsic time of each membrane. The time difference of the stop and start

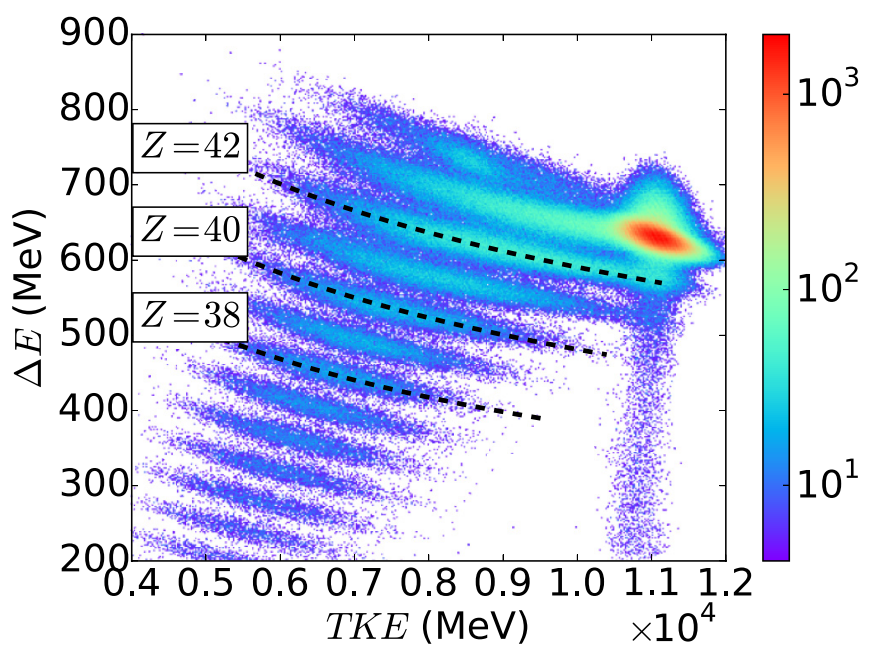

FIG. 3. The $\Delta E-E$ spectrum measured by the LYCCA telescopes for nuclei produced in the secondary reaction of ${ }^{109} \mathrm{Tc}$ ions. It allowed the identification of the proton number of the products. The color bar on the right-hand side indicates the number of counts per bin.

membrane was used for the measurement of the time of flight. From the LYCCA ToF measurement, we calculated the ejectile velocity:

$$
\beta_{\mathrm{LYCCA}}=\frac{L_{2} / \mathrm{ToF}_{\mathrm{LYCCA}}}{c},
$$

with $L_{2}=5.016 \mathrm{~m}$ being the distance between the two membranes. More details on the electronics and the LYCCA detectors can be found in Refs. [23,25]. In addition to the particleidentification, the LYCCA calorimeter tracked the ejectiles combining the positions measured by a DSSSD detector positioned $58 \mathrm{~mm}$ in front of the target and the DSSSD detectors from the telescope positioned $3016 \mathrm{~mm}$ behind the target [25]. The DSSSD detectors were gain matched based on the self-consistent calibration method detailed in Ref. [31]. The CsI crystals were gain matched using the distinct maximum energy deposit by each of the FRS incoming beams. This calibration procedure worked well with the monoenergetic mode of the FRS because each fragment species had a different energy at the final focal plane of the FRS. Nevertheless, this procedure was only applied to the six central LYCCA telescopes with high statistics, corresponding to $85 \%$ of the statistics recorded in this experiment. A bi-dimensional histogram allowed us to determine the proton number of the fragments produced after fragmentation of, e.g., the ${ }^{109} \mathrm{Tc}$ beam (see Fig. 3).

The mass $A$ of the fragments was obtained $[19,25]$ by combining the TKE with the fragment velocity:

$$
A=\frac{\mathrm{TKE}}{u c^{2}(\gamma-1)},
$$

with $u$ being the atomic mass unit, $c$ the speed of light in vacuum, and $\gamma=\frac{1}{\sqrt{1-\beta_{\mathrm{LCCA}}^{2}}}$ the Lorentz factor. The calibration of the LYCCA ToF was essential to improve the mass resolution for the identification of the ejectile fragments. Nevertheless, this calibration was not sufficient to fully resolve 


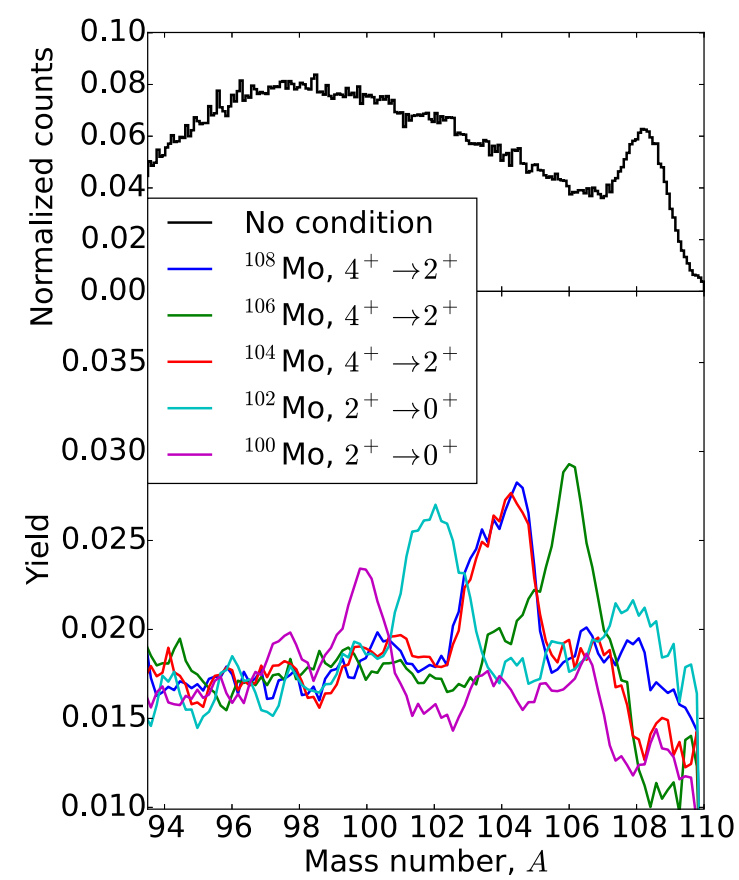

FIG. 4. Mass identification based on the reconstruction of the mass given by Eq. (3). In the upper panel, only a gate on the LYCCA $\triangle E-E$ was applied. For each histogram of the lower panel, two gates were applied, one on the relevant $\gamma$-ray transition (see legend) and one on the molybdenum isotope in the LYCCA $\Delta E-E$ identification histogram in Fig. 3. The yield is the ratio of the number of $\gamma$ rays observed for the energy of the transition over the total number of $\gamma$ rays recorded in an energy range from 200 to $1000 \mathrm{keV}$. The lines corresponding to ${ }^{108} \mathrm{Mo}$ and ${ }^{104} \mathrm{Mo}$ are similar because the energies of the $4^{+} \rightarrow 2^{+}$transition are close.

masses in the region of interest as shown in the upper panel of Fig. 4. Therefore, the known $\gamma$-ray energies of the expected fragments were used to identify their mass. The yields of the different molybdenum fragments, selected by a condition on their Doppler corrected $\gamma$-ray energy, are shown in the lower panel of Fig. 4. This figure shows that the mass resolution achieved was $\sim 1.5$ mass units at full width at half maximum, which was not sufficient to determine the mass of the ejectiles as performed in Refs. [19,25]. Hence, in this analysis we used a mass selection width of 0.8 mass units to reduce the contribution of background and potential contaminants. A summary of the number of events recorded and identified by both FRS and LYCCA is given in Table II. In this table, the events from the two production settings are summed.

The calibration of the 19 AGATA crystals was performed using ${ }^{60} \mathrm{Co}$ and ${ }^{152} \mathrm{Eu}$ sources and followed the standard data-flow treatment of AGATA experiments as described in Refs. [32,33]. Three aspects of the adopted method concerning AGATA data processing are important to stress here. First, a condition was applied on the central-contact energy obtained by the moving window deconvolution algorithm built in the AGATA electronics [18]. This energy had to be the same as the energy derived from the pulse-shape-analysis (PSA) traces recorded over $1-\mu$ s length, which was equivalent to a pile-up rejection [34]. Second, the option assured that the interaction positions obtained with the PSA grid-search algorithm [35] were given by the simulated AGATA Detector Library [36] (ADL) without any smearing of the positions. Third, the AGATA-tracking algorithm [37,38] was not applied. Instead, the energy measured by the germanium central contact was used as the $\gamma$-ray energy, and the $\gamma$-ray hit with the highest energy was chosen as the first interaction position of the $\gamma$ ray inside the germanium detector. This choice, even if counterintuitive for a $\gamma$-tracking array, was driven by the fact that presently the AGATA tracking algorithms assume photon emission at the target position. In our experiments, however, with fragments traveling at half the speed of light a $\gamma$-ray emission could happen up to $3 \mathrm{~cm}$ behind the target position, inducing a tracking inefficiency correlated with the lifetime of the level, which had to be avoided. Even without using a tracking algorithm, the precise determination of the interaction

TABLE II. Number of events identified with the FRS and LYCCA detectors. In this table the number of $\gamma$ rays detected in an energy range of $[200,1000] \mathrm{keV}$ is also given. It is worth noting that the ratio of the number of $\gamma$ rays to the number of ions changes with the selected ion.

\begin{tabular}{|c|c|c|c|c|c|c|c|}
\hline selection & $\begin{array}{c}\text { Number of } \\
\text { identified nuclei }\end{array}$ & $\begin{array}{l}\text { Number of } \\
\text { T10 triggers }\end{array}$ & $\begin{array}{l}\text { Number of } \\
\text { T9 triggers }\end{array}$ & $\begin{array}{c}\text { LYCCA } \\
\text { mass selection }\end{array}$ & $\begin{array}{l}\text { Number of } \\
\text { T10 triggers }\end{array}$ & $\begin{array}{l}\text { Number of } \\
\text { T9 triggers }\end{array}$ & $\begin{array}{c}\text { Number of } \\
\gamma \text { events }\end{array}$ \\
\hline \multirow{4}{*}{${ }^{109} \mathrm{Tc}$} & \multirow{4}{*}{$9.34 \times 10^{6}$} & \multirow{4}{*}{$4.94 \times 10^{4}$} & \multirow{4}{*}{$3.13 \times 10^{6}$} & Any & 1676 & $2.04 \times 10^{5}$ & $2.86 \times 10^{5}$ \\
\hline & & & & 108 & 145 & $8.64 \times 10^{4}$ & $1.85 \times 10^{3}$ \\
\hline & & & & 106 & 27 & $3.00 \times 10^{4}$ & $2.34 \times 10^{3}$ \\
\hline & & & & 100 & 37 & $4.48 \times 10^{4}$ & $4.94 \times 10^{3}$ \\
\hline \multirow{4}{*}{${ }^{108} \mathrm{Mo}$} & \multirow{4}{*}{$4.22 \times 10^{6}$} & \multirow{4}{*}{$6.40 \times 10^{4}$} & \multirow{4}{*}{$3.94 \times 10^{6}$} & Any & 15309 & $9.80 \times 10^{5}$ & $4.30 \times 10^{5}$ \\
\hline & & & & 108 & 4516 & $2.62 \times 10^{5}$ & $1.25 \times 10^{4}$ \\
\hline & & & & 106 & 261 & $1.98 \times 10^{4}$ & $6.78 \times 10^{4}$ \\
\hline & & & & 104 & 122 & $1.14 \times 10^{4}$ & $7.84 \times 10^{4}$ \\
\hline
\end{tabular}


position by the AGATA PSA algorithm was essential to perform the Doppler correction.

A time offset was added to the LYCCA ToF to determine the optimum ejectile velocity for the Doppler correction. This offset was determined before the experiment minimizing the width of the uranium $\mathrm{x}$ rays after Doppler correction [39], and verified with the transitions of ${ }^{104} \mathrm{Mo}$. Because this offset depends only on the cable length, the same offset was used in this work for the Doppler correction of the $\gamma$ rays emitted by the molybdenum isotopes. The emission angle was calculated combining the interaction position of the $\gamma$-ray hit corresponding to the highest energy deposited in a given germanium detector with the positions of the fragments measured by the two FRS TPCs and the LYCCA DSSSDs. The peak-to-background $(P / B)$ ratio was improved [40] from $1.6 \%$ to $3.2 \%$ for the $4^{+}$to $2^{+}$transition of ${ }^{104} \mathrm{Mo}$ by setting a 40-ns-wide time window on the prompt peak, selecting only events with a $\gamma$-ray multiplicity smaller than four, and discarding events with multiple hits in any of the particle-tracking detectors.

The $\gamma$-ray spectra obtained for the even-even molybdenum isotopes are shown in Fig. 5. Only the transitions belonging to these nuclei are indicated by the green dashed lines. The

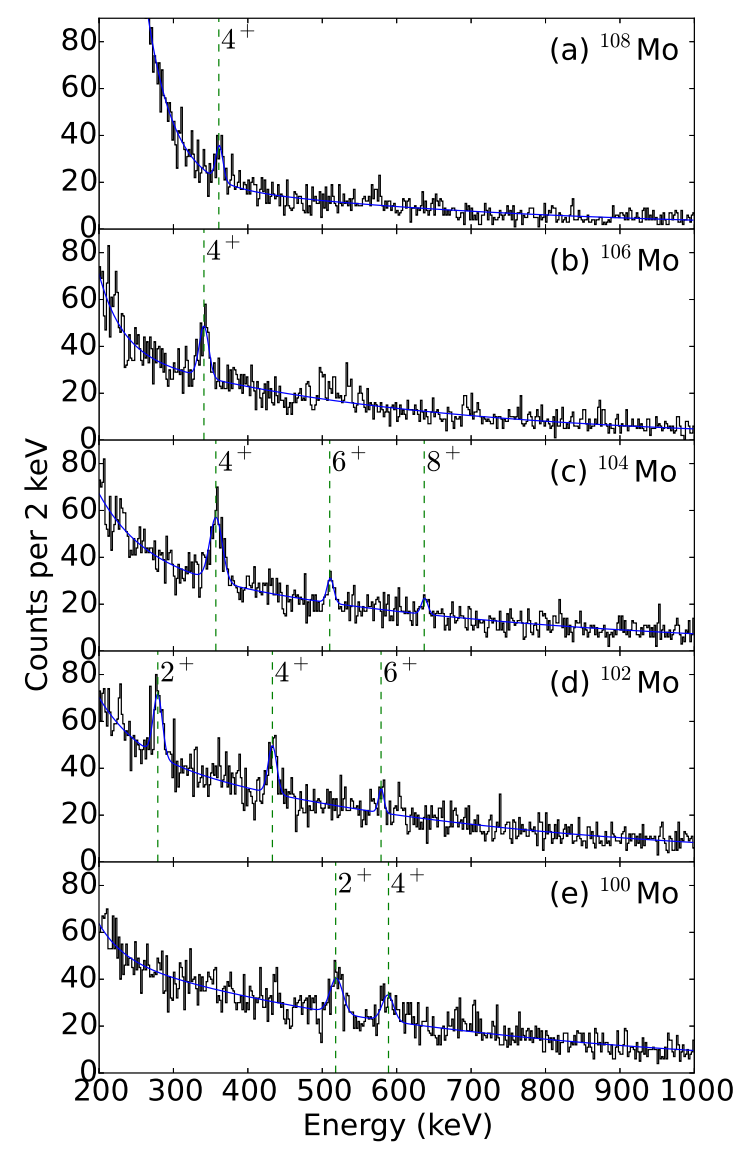

FIG. 5. Observed transitions in even-even molybdenum isotopes. The blue curves correspond to the model fitted on the data and used to determine the mean energy of the observed transitions. The green dashed lines indicate the mean energy of the observed transitions as given in the fourth column of Table III. other peaks belong to odd-mass molybdenum isotopes. No transitions from the odd-mass nuclei were found with the same energy as the transitions from the even-mass nuclei. A resolution of $\sim 10.5_{-2.5}^{+3.0} \mathrm{keV}$ at FWHM was achieved at $511 \mathrm{keV}$, measured for the $6^{+}$to $4^{+}$transition of ${ }^{104} \mathrm{Mo}$. This resolution is consistent with the $\sim 5$-mm position resolution obtained by the PSA algorithm [41].

\section{LIFETIME DETERMINATION}

At the secondary target, where the isotopes of interest were produced, the beam velocity was $\beta \sim 0.5$. The mean lifetime of the $2^{+}, 4^{+}$, and $6^{+}$states of the even-even ${ }^{100-108} \mathrm{Mo}$ range from about 2 to $200 \mathrm{ps}$. Thus the mean value of the decay position was located between 0.3 and $30 \mathrm{~mm}$ from the excitation point. Experimentally, the exact de-excitation position information was not accessible, and for the Doppler correction we assumed that the decay occurred in the middle of the target. In general, the real emission angle $\left(\theta_{\mathrm{em}}\right)$ of the photon was therefore different from the one that was used for the Doppler correction $\theta_{\mathrm{exp}}$. The Doppler corrected energy $E_{\mathrm{DC}}$ of the $\gamma$ rays emitted in-flight relates to the measured energy $E_{\text {shift }}$ as

$$
E_{\mathrm{DC}}=E_{\text {shift }} \frac{1-\beta \cos \left(\theta_{\mathrm{exp}}\right)}{\sqrt{1-\beta^{2}}} .
$$

A wrong emission angle thus leads to an "imprecise" Doppler correction, with a shift with respect to the rest energy $E_{0}$ that is a function of the excited state mean lifetime. This centroid shift is defined as the ratio:

$$
R_{\text {shift }}=\frac{E_{0}-E_{\mathrm{DC}}}{E_{0}}
$$

is, hence, sensitive to the level lifetime. This method is applicable only for transitions with a well-known energy $E_{0}$ which was the case for the experiment described in this paper.

The relation between the mean lifetime and the energy shift was determined using Monte Carlo (MC) simulations following a similar methodology as the one described in Refs. [27,42]. Because the reaction cross sections at the secondary target are independent of the beam energy [43], we assumed an excitation at the center of the target. This assumption simplified the model but did not affect the result because we only considered the centroid shift $R_{\text {shift }}$. For a given mean lifetime of a state and for each MC event, a random decay position of the level was simulated. At each decay position, the particle velocity was determined using the Ziegler estimate of the slowing-down process [44]. The Doppler shift was calculated with an angle of detection chosen randomly for each event with a probability distribution that followed the experimental detection angles. A Doppler correction was then performed assuming a decay at the target center for both $\gamma$-ray emission angle and ion velocity, as in the experimental data analysis. This produced the curves shown in Fig. 6.

If a direct feeding was observed, the measured intensity ratio of the involved transitions was used to calculate the decay position. For example, the $6^{+}$state of ${ }^{104} \mathrm{Mo}$ was fed by the $8^{+}$ state with $10 \%$ probability (see Table III). The $\gamma$ ray emitted from the $6^{+}$has a probability of 0.1 to be emitted after the 


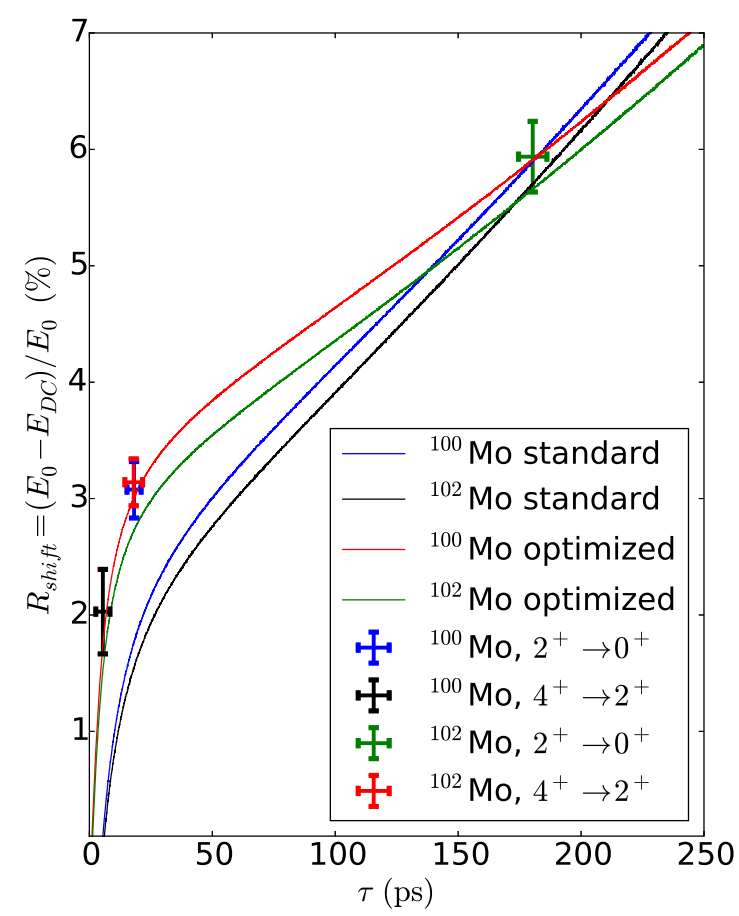

FIG. 6. The curves correspond to the simulated values of $R_{\text {shift }}$ as a function of $\tau$, the mean lifetime. The data points for ${ }^{100,102}$ Mo correspond to previous measurements [45]. In the legend "standard" stands for assumed velocity and target thickness, while "optimized" corresponds to the set of parameters $\left(t_{\text {thick }}, f\right)=$ $\left(556(13) \mathrm{mg} / \mathrm{cm}^{2}, 0.916(19)\right)$ for which the simulations were consistent with known lifetimes (see text for details). For clarity, the data points of ${ }^{104} \mathrm{Mo}$ and feeding simulations are not shown in this plot, but they were taken into account in the determination of $\left(t_{\text {thick }}, f\right)$. decay of the $8^{+}$, thus farther away from the production point than in the case of no feeding. The mean lifetime of the feeder was first determined by our simulations and then taken into account in the simulations for the states fed. The number of events in the Monte Carlo simulations was increased until convergence of the lifetime was reached at a precision of a tenth of a picosecond. The unobserved feeding was not taken into account in the simulations.

The measured dependence of the ratio $R$ to the mean lifetime of an excited state are given in Table III. Nevertheless, taking the measured beam velocity and the assumed target thickness it was not possible to fully reproduce the trend nor the absolute value of the ratio $R(\tau)$. The comparison of the simulations for the nominal target thickness and measured beam velocity are shown in Fig. 6.

The observed differences between the simulated and experimentally determined $R$ values (given in Table III) for $\gamma$-ray transitions with known lifetimes were suspected to be because of differences between the assumed and actual target thickness and ion velocity. Hence, the previously measured mean lifetimes of the observed excited states of ${ }^{100,102,104} \mathrm{Mo}$ (listed in Table III) were used to determine the effective target thickness $\left(t_{\text {thick }}\right)$ and the velocity ratio $(f)$ defined as

$$
f=\beta_{\text {em }} / \beta_{\exp }
$$

with $\beta_{\exp }$ the velocity used experimentally for the Doppler correction and $\beta_{\text {em }}$ the velocity of the ejectiles. To determine the inherent error on the evaluation of these two parameters we constructed the likelihood $\mathcal{L}$ defined as

$$
\mathcal{L}\left(t_{\text {thick }}, f\right)=p\left(R_{\text {sim }}\left(\tau ; t_{\text {thick }}, f\right)\right) \times p\left(R_{\text {exp }}\right) \times p(\tau)
$$

TABLE III. Summary of the mean lifetimes $\tau$ measured in the present work. The relative yields correspond to the ratio of the peak area of the transition of interest over the sum of the peak area of the observed transitions. The lifetime uncertainties indicated by $\sigma_{\text {stat }}$ and $\sigma_{\text {syst }}$ in this table are given by the 18th and 64th percentile of the a posteriori likelihood distribution. The definition of the content of the columns is given

\begin{tabular}{|c|c|c|c|c|c|c|c|c|c|c|c|}
\hline Nucleus & $I_{i} \rightarrow I_{f}$ & $\begin{array}{c}E_{0}[45] \\
(\mathrm{keV})\end{array}$ & $\begin{array}{c}E_{\mathrm{DC}} \\
(\mathrm{keV})\end{array}$ & $\begin{array}{l}\text { Ratio } \\
R(\%)\end{array}$ & $\begin{array}{c}\text { Relative } \\
\text { yields }(\%)\end{array}$ & $\begin{array}{c}\tau \\
(\mathrm{ps})\end{array}$ & $\begin{array}{l}\sigma_{\text {stat }} \\
(\mathrm{ps})\end{array}$ & $\begin{array}{l}\sigma_{\text {syst }} \\
(\mathrm{ps})\end{array}$ & $\begin{array}{l}\sigma_{\text {tot }} \\
(\mathrm{ps})\end{array}$ & $\begin{array}{l}\left|Q_{0}^{t}\right| \\
(\mathrm{eb})\end{array}$ & $\begin{array}{c}\text { Literature value } \\
\tau(\mathrm{ps})\end{array}$ \\
\hline${ }^{108} \mathrm{Mo}$ & $4^{+} \rightarrow 2^{+}$ & 370.9 & $361.7_{-1.0}^{+1.0}$ & $2.47_{-0.27}^{+0.26}$ & 100 & 29.7 & ${ }_{-9.1}^{+11.3}$ & $\begin{array}{l}+2.3 \\
-1.9\end{array}$ & ${ }_{-9.3}^{+11.5}$ & $3.7_{-0.6}^{+0.7}$ & - \\
\hline${ }^{104} \mathrm{Mo}$ & $4^{+} \rightarrow 2^{+}$ & 368.4 & $357.3_{-0.9}^{+0.9}$ & $3.01_{-0.24}^{+0.24}$ & 74 & 31.8 & ${ }_{-9.4}^{+12.4}$ & $\begin{array}{l}+3.7 \\
-3.9\end{array}$ & $\begin{array}{l}+13.3 \\
-10.2\end{array}$ & $3.7_{-0.6}^{+0.7}$ & $37.7 \pm 1.1 \bullet^{\mathrm{b}}$ \\
\hline${ }^{104} \mathrm{Mo}$ & $6^{+} \rightarrow 4^{+}$ & 519.2 & $510.9_{-1.2}^{+1.2}$ & $1.60_{-0.23}^{+0.24}$ & 16 & 5.3 & ${ }_{-1.1}^{+1.6}$ & $\begin{array}{l}+0.6 \\
-0.4\end{array}$ & $\begin{array}{l}+1.7 \\
-1.2\end{array}$ & $3.6_{-0.4}^{+0.4}$ & $6.83 \pm 0.21 \diamond^{b}$ \\
\hline${ }^{102} \mathrm{Mo}$ & $2^{+} \rightarrow 0^{+}$ & 296.6 & $279.0_{-0.9}^{+0.9}$ & $5.94_{-0.30}^{+0.30}$ & 50 & 186.9 & $\begin{array}{l}+17.4 \\
-17.8\end{array}$ & $\begin{array}{l}+5.6 \\
-5.6\end{array}$ & $\begin{array}{l}+18.3 \\
-18.7\end{array}$ & $3.1_{-0.1}^{+0.2}$ & $180 \pm 6>^{c}$ \\
\hline${ }^{102} \mathrm{Mo}$ & $4^{+} \rightarrow 2^{+}$ & 447.1 & $433.1_{-0.9}^{+0.9}$ & $3.14_{-0.20}^{+0.20}$ & 38 & 27.8 & $\begin{array}{l}+9.0 \\
-6.7\end{array}$ & $\begin{array}{l}+5.5 \\
-4.5\end{array}$ & ${ }_{-8.1}^{+10.5}$ & $2.4_{-0.3}^{+0.4}$ & $18.0 \pm 3.6{ }^{\mathrm{d}}$ \\
\hline${ }^{102} \mathrm{Mo}$ & $6^{+} \rightarrow 4^{+}$ & 584.2 & $579.0_{-1.5}^{+1.5}$ & $0.89_{-0.25}^{+0.25}$ & 12 & 3.2 & $\stackrel{+}{-0.7}^{+0.7}$ & $\begin{array}{l}+0.2 \\
-0.2\end{array}$ & $\stackrel{+}{-0.7}^{+0.7}$ & $3.5_{-0.3}^{+0.5}$ & - \\
\hline${ }^{100} \mathrm{Mo}$ & $2^{+} \rightarrow 0^{+}$ & 535.6 & $519.1_{-1.3}^{+1.3}$ & $3.08_{-0.24}^{+0.24}$ & 60 & 14.8 & $\stackrel{+}{+4.1}_{-6.1}$ & $\begin{array}{l}+4.0 \\
-2.8\end{array}$ & $\begin{array}{l}+7.3 \\
{ }_{-5.0}\end{array}$ & $2.5_{-0.4}^{+0.4}$ & $18.2 \pm 2.9 \gamma^{\mathrm{e}}$ \\
\hline
\end{tabular}
in the text (see Sec. IV). Lifetimes indicated by were used for normalization.

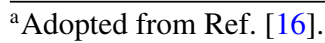

bAdopted from Ref. [47].

${ }^{\mathrm{c}}$ Adopted from Ref. [48].

${ }^{\mathrm{d}}$ Adopted from Ref. [46].

eAdopted from Ref. [49].

${ }^{\mathrm{f}}$ Adopted from Ref. [50].
} 
where $p(\tau)$ is the probability of the previously measured value and $p\left(R_{\exp }\right)$ the probability of the measured ratio. The parameters $\left(t_{\text {thick }}, f\right)=\left(556(13) \mathrm{mg} / \mathrm{cm}^{2}, 0.916(19)\right)$ maximize the likelihood for the known mean lifetimes of the excited states of ${ }^{100,102,104}$ Mo. A correlation coefficient of $\rho_{\left(t_{\text {thick }}, f\right)}=0.32$ was obtained between the two parameters. The optimization is illustrated in Fig. 6 for ${ }^{100,102}$ Mo isotopes. The average velocity, $\left(<\beta_{\exp }>\sim 0.48\right)$, corresponding to the minimum width after Doppler correction of the transition is $8.4 \%$ larger than the actual average velocity of the flying ejectile $\left(<\beta_{\text {em }}>\sim 0.440(6)\right)$. The effective target thickness is $21 \%$ smaller than expected.

A second method was also used to estimate the ion velocity. As shown in Eq. (4) the Doppler shifted energy at a given angle depends only on the beam velocity. Thus, the beam velocity from the experimental data was determined by solving the second-order polynomial equation in $\beta$. Using the $2^{+}$to $0^{+}$ transition of ${ }^{102} \mathrm{Mo}$, this method yielded [40] a beam velocity of $\beta_{\text {em }}=0.439$ (13), which is in full agreement with the value of $0.440(6)$ obtained from the minimization procedure described above.

The fact that the effective target thickness is smaller than expected was explained by a slow-down power that was not fully described by the simulations. A smaller target thickness corresponded to a larger stopping power than given in Ref. [44].

The uncertainties obtained for the effective target thickness and on the velocity fraction were the main source of systematic errors in this measurement which are given by $\sigma_{\text {syst }}$ in Table III. The sum of the two uncertainties, $\sigma_{\text {tot }}$, was defined as $\sigma_{\text {tot }}=$ $\sqrt{\sigma_{\text {stat }}^{2}+\sigma_{\text {syst }}^{2}}$.

A summary of this measurement for the molybdenum isotopes is given in Table III. We note that the $4^{+}$to $2^{+}$ transition in ${ }^{102}$ Mo is just in agreement with the literature value from Ref. [46]. A possible reason could be the existence of a feeder state that cannot be determined in this experiment. Nevertheless, even if this value was used for the minimization of the parameters [see Eq. (7)], the systematic errors take into account the uncertainties that might arise from the procedure developed here. Furthermore, the lifetime determined in this work and the literature values of ${ }^{106} \mathrm{Mo}$ are within one standard deviation is a proof of the robustness of the procedure because the value was not used for the minimization. In addition, two new mean lifetimes, the $6^{+}$of ${ }^{102} \mathrm{Mo}$ and the $4^{+}$of ${ }^{108} \mathrm{Mo}$, have been determined in this work.

\section{DISCUSSION}

In Fig. 7 the evolution of the transitional quadrupole moments $Q_{0}^{t}\left(I_{i}\right)$ is plotted as a function of the mass of the molybdenum isotopes. For the first excited $2^{+}$state, it seems that there is a gradual increase of the quadrupole moment with the neutron number. Nevertheless, this conclusion could be different considering the transitional quadrupole moment of the 4 state, that seems to decrease at $N=66$. This decrease of collectivity is also suggested by the Grodzins relation [51] and the value of the $2_{1}^{+}$energy [45]. Similarly, maximum transition probabilities were also observed for the zirconium isotopes [52].

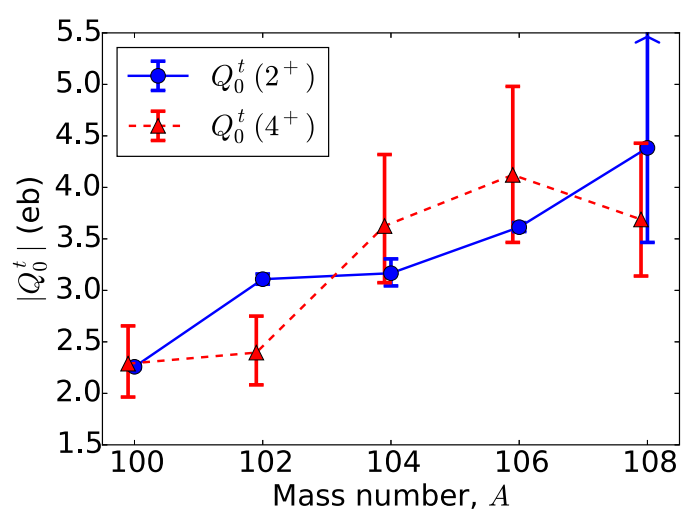

FIG. 7. Absolute value of transitional quadrupole moments as a function of the mass of the neutron-rich molybdenum isotopes. Some of the values are slightly displaced on the $x$ axis for clarity. The $Q_{0}^{t}\left(4^{+}\right)$shown in this plots are extracted from this work. Instead the $Q_{0}^{t}\left(2^{+}\right)$used the literature values (see Table III). The arrow (for the $2^{+}$state of ${ }^{108} \mathrm{Mo}$ ) indicates that the previous lifetime measurement [53] has an error bigger than displayed here.

To explain the structure of the light molybdenum isotope BMF calculations within the SCCM framework [15] with the Gogny D1S functional [14] have been carried out. In this method, the nuclear states are obtained by linear combinations of particle number and angular momentum projected states with different axial and nonaxial shapes. Both the intrinsic states and the coefficients of the linear combinations are obtained self-consistently from the same underlying interaction (for details see Ref. [15]).

In Fig. 8, the calculated energies for the $2^{+}, 4^{+}$, and $6^{+}$states of the ground-state band are compared with the experimental values. The trend of the experimental data is nicely reproduced by the present calculations, although the values are systematically larger. This stretching is expected because of the lack of cranking states in the set of mean-fieldlike wave functions used to perform the SCCM calculations [54]. The inclusion of such a degree of freedom would lead to

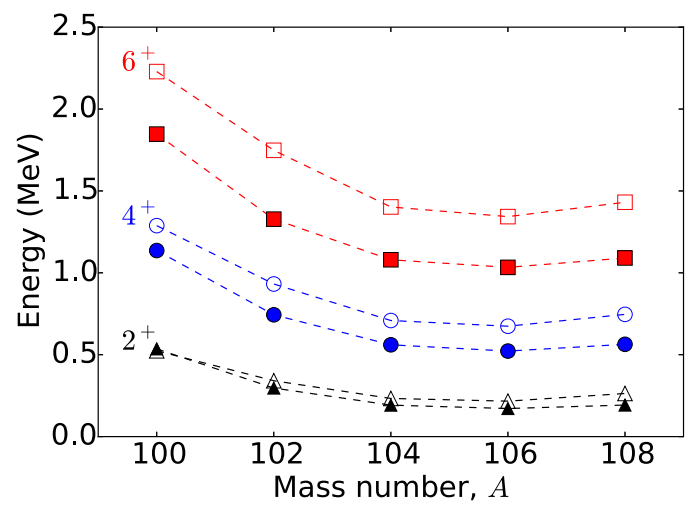

FIG. 8. Comparison of the energies of the ground-state band members (open symbols) calculated with beyond mean-field calculation [15] with the literature value [45] (filled symbols). Dashed lines are used to guide the eye. Some of the values are slightly displaced on the $x$ axis for clarity. 


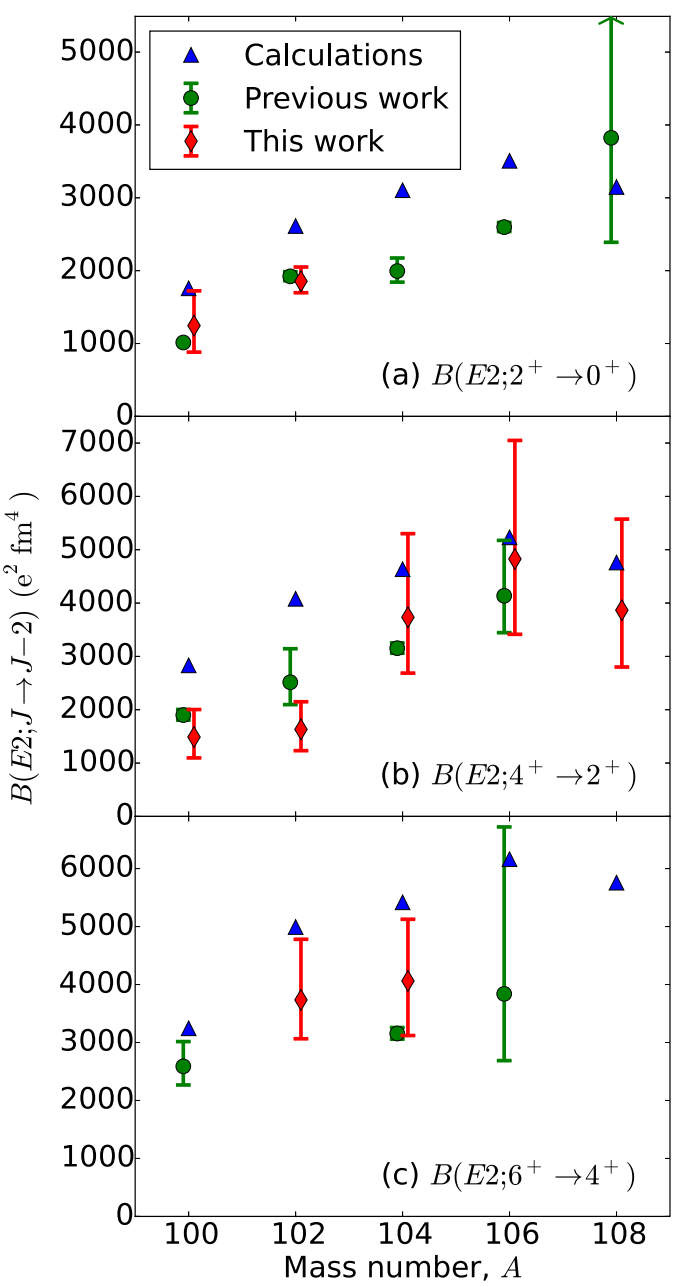

FIG. 9. Comparison of reduced transition strengths measured in this work with the experimental literature values (see Table III) and with those obtained from BMF calculations. Because low-spin states were preferably populated in the present experiment, only levels up to $6^{+}$are displayed. The arrow (for the $2^{+}$state of ${ }^{108} \mathrm{Mo}$ ) indicates that the previous lifetime measurement [53] has an error bigger than displayed here.

a better quantitative agreement with the experimental values at the price of a prohibitive increase of the computational burden [55].

Similarly, the reduced transition strengths calculated with the SCCM method are systematically larger than the experimental results as shown in Fig. 9. This excess could be again partially corrected by including cranking terms [56] but could also indicate an overestimation of the deformation by the present Gogny functional. It is worth mentioning that no effective charges are used in this approach. The present calculations predict an increase of the $B(E 2)$ values in the ground-state band from ${ }^{100} \mathrm{Mo}$ to ${ }^{106} \mathrm{Mo}$, where a maximum is reached, and a slight decrease in ${ }^{108} \mathrm{Mo}$.

The overall experimental trend of the reduced transition strength, from previous work and from the newly measured lifetimes presented here, is well reproduced by the BMF calculations (see Fig. 9). There are only two discrepancies between these calculations and the data, namely, the
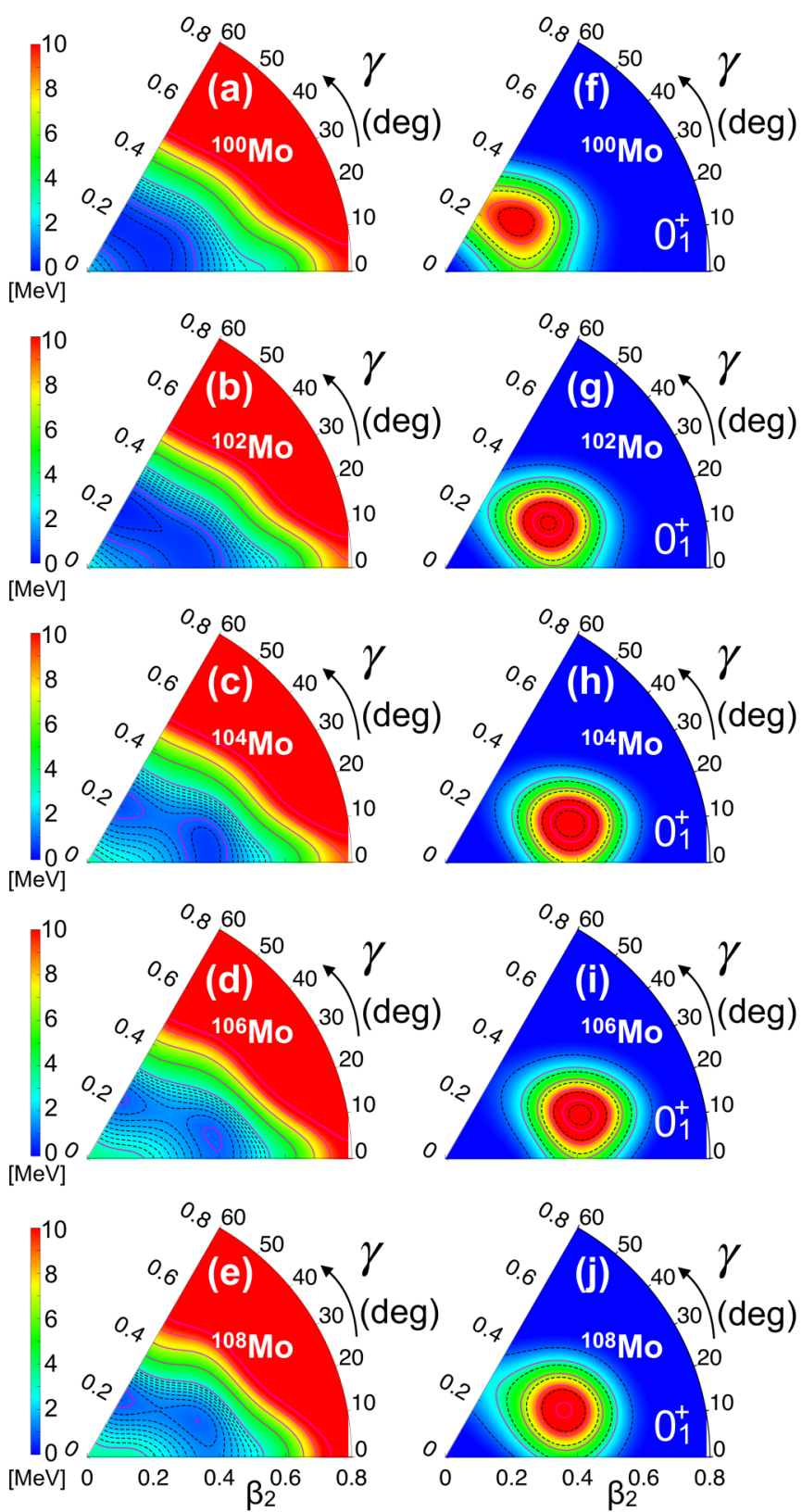

FIG. 10. (a)-(e) Particle-number projected potential energy surfaces-normalized to the minimum of each surface contour line, separated by $0.25 \mathrm{MeV}$ (dashed) and $2 \mathrm{MeV}$ (continuous)—and (f)-(j) SCCM collective wave functions-normalized to one, reddish means large and blueish small— for ${ }^{100,102,104,106,108}$ Mo isotopes calculated with the Gogny D1S interaction.

$B\left(E 2 ; 2^{+} \rightarrow 0^{+}\right)$in ${ }^{108} \mathrm{Mo}[53]$ and the $B\left(E 2 ; 6^{+} \rightarrow 4^{+}\right)$in ${ }^{106}$ Mo [16]. Nevertheless, these lifetime measurements have very large error bars.

The collective nature of these nuclei can be studied within the SCCM by analyzing first the potential energy surfaces (PES) and, second, the collective wave functions. The latter represent the weights of the different intrinsic shapes in each nuclear state.

In Fig. 10 (left panel) the particle-number projected energy as a function of the quadrupole deformation $\left(\beta_{2}, \gamma\right)$ is repre- 
sented for the nuclei ${ }^{100,102,104,106,108} \mathrm{Mo}$. In general, these PES show a noticeable $\gamma$ softness, with almost degenerate states in a lane approximately $\beta_{2} \approx 0.3$ wide along the gamma direction that connects prolate $\left(\beta_{2} \approx 0.35, \gamma=0^{\circ}\right)$ and oblate $\left(\beta_{2} \approx\right.$ $0.2, \gamma=60^{\circ}$ ) states. Nevertheless, the nuclei ${ }^{100,102}$ Mo are a bit less deformed, and the PES of the isotopes ${ }^{104,106,108} \mathrm{Mo}$ show two minima, one oblate, and the other moves from prolate $\left({ }^{104} \mathrm{Mo}\right)$ to pure triaxial configurations $\left({ }^{108} \mathrm{Mo}\right)$.

After performing the angular momentum projection and the configuration (shape) mixing, the collective wave functions for the individual states can be evaluated. The calculations predict for all nuclei studied here a structure consisting of triaxial rotational ground-state (g.s.) bands, $\gamma$ bands associated with them, and second excited axial rotational bands built on top of the $0_{2}^{+}$. The latter have a prolate character for ${ }^{100,102} \mathrm{Mo}$ and an oblate character for ${ }^{104,106,108} \mathrm{Mo}$. In the right panel of Fig. 10 the ground-state collective wave functions are plotted. All of them have their maxima at pure triaxial configurations. Starting from the lightest, the deformation of the ground state evolves from smaller $\beta_{2}$ deformations closer to the oblate axis towards larger deformations with a more prolate character $\left(\gamma<30^{\circ}\right)$ for ${ }^{102,104,106,108} \mathrm{Mo}$. In fact, these isotopes are somewhat similar and this is the reason why the changes in the excitation energies (Fig. 8) are so mild. Finally, the effect of the angular momentum restoration and configuration mixing is observed by comparing the potential wells on the left of Fig. 10 and the final distribution of the ground states on the right. Eventually, the prolate configuration was shifted towards a more triaxial character in ${ }^{104,106,108} \mathrm{Mo}$.

In a recent paper, the spectroscopic moments of the $2_{1}^{+}$and $2_{2}^{+}$states and the reduced transition probabilities between these states and the $0_{1}^{+}$and $0_{2}^{+}$states have been measured in ${ }^{96,98} \mathrm{Sr}$ [57]. This data suggests the presence of shape coexistence between spherical and prolate deformed states with a shape inversion from a spherical to a prolate ground-state band at $N=60$. However, in the case of the molybdenum isotopes the situation is different, i.e., the ground-state collective wave functions for $N=58$ and $N=60$ show a somewhat smooth transition from triaxial shapes with a more oblate character $\left({ }^{100} \mathrm{Mo}\right)$ towards a more prolate character $\left({ }^{102} \mathrm{Mo}\right)$ without any signature of a shape inversion.

\section{CONCLUSIONS}

In summary, the work described in this paper reports the first experiment where a relativistic Doppler-shift-attenuation method was applied for the first time to products of a two-step reaction. The high-resolution prompt $\gamma$-ray spectroscopy experiment was made possible by the combination of FRS, LYCCA, and AGATA. Lifetimes in the range $\tau \in$
$[2,250]$ ps were measured for several excited states in five neutron-rich even-even molybdenum isotopes in one single experiment.

This paper reports two previously unknown lifetimes: that of the $4^{+}$state of ${ }^{108}$ Mo with $\tau=29.7_{-9.1}^{+11.3} \mathrm{ps}$, and the $6^{+}$state of ${ }^{102}$ Mo with $\tau=3.2_{-0.7}^{+0.7}$ ps.

In this midshell mass $A \approx 100$ region previous measurements indicate a maximum deformation for $N=64$ for zirconium $(Z=40)$ and ruthenium $(Z=44)$ isotopes $[52,58,59]$. This work provides an indication that a maximum deformation is reached at $N=64$ for the molybdenum isotopes as well. Furthermore, the lifetime measurement of the $4^{+}$excited state of ${ }^{108}$ Mo suggests a decrease of the transition probability after $N=64$. BMF calculations within the SCCM method with the Gogny D1S functional reproduce nicely the trends of the experimental values although both the excitation energies and transition probabilities are overestimated. This problem could be corrected by including cranking terms in the theoretical framework or it could be an indication of an overestimation of the deformation by the Gogny functional. Calculations also show that these nuclei are triaxially deformed, showing the relevance of this degree of freedom in this region of the nuclear chart.

The experimental method described here opens new perspectives for lifetime measurements in nuclei far from stability once in-beam $\gamma$-ray spectroscopy become possible within the HiSPEC project [60] using RIB beams delivered by the Super-FRS [61] at the future FAIR facility.

\section{ACKNOWLEDGMENT}

The authors are thankful to the FRS team, and the PreSPEC and AGATA collaborations for their effort in setting up the experiments at GSI. The authors thank M. Fouesneau for the discussions of the Bayesian data analysis methods that was used in this work. The Monte Carlo-Markov-Chain python package described in Ref. [62] together with the GSI computing center was intensively used throughout this work. This work was supported by the BMBF under Grants No. 05P09RDFN4, No. 05P12RDFN8, and No. 05P12PKFNE and by the LOEWE center HIC for FAIR. Part of this work was supported by the P2IO excellence center. This work was also supported by the European Community FP7-Capacities, ENSAR Contract No. 262010, by the Swedish Research Council under Contracts No. 2010-147, No. 2010-3694, No. 2011-5253, No. 2011-6127, and No. 2014-5558, by the IN2P3GSI No. 02-42 collaboration, by the Science and Technology Facilities Council (STFC) and by MINECO, Spain, under Grant No. FPA2014-57196-C5, and Generalitat Valenciana, Spain, under Grant No. PROMETEOII/2014/019.
[1] S. A. E. Johansson, Nucl. Phys. A 64, 147 (1965).

[2] R. Rodríguez-Guzmán et al., Phys. Lett. B 691, 202 (2010).

[3] F. R. Xu, P. M. Walker, and R. Wyss, Phys. Rev. C 65, 021303 (2002).

[4] K. Wrzosek-Lipska et al., Phys. Rev. C 86, 064305 (2012).
[5] A. G. Smith, J. L. Durell, W. R. Phillips, W. Urban, P. Sarriguren, and I. Ahmad, Phys. Rev. C 86, 014321 (2012).

[6] H. Watanabe et al., Phys. Lett. B 704, 270 (2011).

[7] J. A. Pinston et al., Phys. Rev. C 74, 064304 (2006).

[8] H. Hua et al., Phys. Rev. C 69, 014317 (2004). 
[9] F. Iachello et al., The Interacting Boson Model (Cambridge University Press, Cambridge, 1987).

[10] R. F. Casten, Nat. Phys. 2, 811 (2006).

[11] K. W. Schmid et al., Rep. Prog. Phys. 50, 731 (1987).

[12] J. P. Delaroche et al., Phys. Rev. C 81, 014303 (2010).

[13] D. Rowe, Nuclear Collective Motion: Models and Theory (World Scientific, Singapore, 2010).

[14] J. Berger et al., Nucl. Phys. A 428, 23 (1984).

[15] T. R. Rodríguez and J. L. Egido, Phys. Rev. C 81, 064323 (2010).

[16] C. Hutter et al., Phys. Rev. C 67, 054315 (2003).

[17] A. Guessous et al., Phys. Rev. C 53, 1191 (1996).

[18] S. Akkoyun et al., Nucl. Instrum. Methods A 668, 26 (2012).

[19] N. Pietralla et al., EPJ Web of Conferences 66, 02083 (2014).

[20] W. Henning, Nucl. Phys. A 538, 637 (1992).

[21] H. Geissel et al., Nucl. Instrum. Methods B 70, 286 (1992).

[22] B. Jurado et al., Nucl. Instrum. Methods A 483, 603 (2002).

[23] D. Ralet et al., Nucl. Instrum. Methods A 786, 32 (2015).

[24] H. Wollersheim et al., Nucl. Instrum. Methods A 537, 637 (2005).

[25] P. Golubev et al., Nucl. Instrum. Methods A 723, 55 (2013).

[26] A. Giaz et al., Nucl. Instrum. Methods A 729, 910 (2013).

[27] C. Domingo-Pardo et al., Nucl. Instrum. Methods A 694, 297 (2012).

[28] A. Stolz et al., Phys. Rev. C 65, 064603 (2002).

[29] V. Hlinka et al., Nucl. Instrum. Methods A 419, 503 (1998).

[30] R. Hoischen et al., Nucl. Instrum. Methods A 654, 354 (2011).

[31] M. Reese et al., Nucl. Instrum. Methods A 779, 63 (2015).

[32] N. Lalović et al., Nucl. Instrum. Methods A 806, 258 (2016).

[33] N. Lalović et al., EPJ. Web of Conferences 93, 07007 (2015).

[34] T. Petrovic et al., Nuclear Science, IEEE Transactions on 61, 584 (2014).

[35] R. Venturelli et al., LNL Annual Report 204, 220 (2004).
[36] B. Bruyneel et al., Eur. Phys. J. A 52, 11 (2016).

[37] A. Lopez-Martens et al., Nucl. Instrum. Methods A 533, 454 (2004).

[38] D. Bazzacco, Nucl. Phys. A 746, 248 (2004).

[39] D. Ralet et al., in GSI Report, Vol. 2015-1 (GSI, Darmstadt, 2015), pp. 157.

[40] D. Ralet, Ph.D thesis, Technische Universität, Darmstadt, 2015.

[41] F. Recchia et al., Nucl. Instrum. Methods A 604, 555 (2009).

[42] P. Doornenbal et al., Nucl. Instrum. Methods A 613, 218 (2010).

[43] K. Sümmerer, Phys. Rev. C 86, 014601 (2012).

[44] J. F. Ziegler, Handbook of Stopping Cross-sections for Energetic Ions in All Elements (Pergamon Press, Oxford, 1980).

[45] Evaluated Nuclear Structure Data File, National Nuclear Data Center, International Atomic Energy Agency, Vienna.

[46] H. Bohn et al., Z. Phys. A: At. Nucl. 274, 327 (1975).

[47] A. G. Smith et al., J. Phys. G 28, 2307 (2002).

[48] M. Liang et al., Z. Phys. A 340, 223 (1991).

[49] M. Paradis et al., Phys. Rev. C 14, 835 (1976).

[50] M. Pignanelli et al., Nucl. Phys. A 540, 27 (1992).

[51] L. Grodzins, Phys. Lett. 2, 88 (1962).

[52] F. Browne et al., Phys. Lett. B 750, 448 (2015).

[53] H. Penttilä et al., Phys. Rev. C 54, 2760 (1996).

[54] M. Borrajo et al., Phys. Lett. B 746, 341 (2015).

[55] Tomás R. Rodríguez, Eur. Phys. J. A 52, 190 (2016).

[56] M. Borrajo et al., Acta. Phys. Pol. B Proc. Suppl. 8, 567 (2015).

[57] E. Clément et al., Phys. Rev. Lett. 116, 022701 (2016).

[58] A. Görgen et al., J. Phys. G 43, 024002 (2016).

[59] L. Grente et al., EPJ Web Conf. 62, 01002 (2013).

[60] Z. Podolyák, Nucl. Instrum. Methods B 266, 4589 (2008).

[61] H. Geissel et al., Nucl. Instrum. Methods B 204, 71 (2003).

[62] D. Foreman-Mackey et al., Publications of the Astronomical Society of the Pacific 125, 306 (2013). 